\title{
Interpretation of Be migration studies at JET and validation of an integrated numerical model for plasma impurity transport and wall composition dynamics
}

\author{
M. Reinelt ${ }^{\mathrm{a}^{*}}$, K. Krieger ${ }^{\mathrm{a}}$, S. Lisgo ${ }^{\mathrm{c}}$, K. Schmid ${ }^{\mathrm{a}}$, S. Brezinsek ${ }^{\mathrm{b}}$, JET EFDA Contributors ${ }^{\mathrm{d}}$ \\ a Max-Planck Institut für Plasmaphysik, EURATOM Association, 85748 Garching, Germany. \\ ${ }^{b}$ Forschungszentrum Jülich, 52425 Jülich, Germany. \\ ${ }^{c}$ ITER Organisation, Fusion Science and Technology Department, 13067 Saint Paul Lez Durance \\ Cedes, France. \\ ${ }^{d} J E T-E F D A$, Culham Science Centre, OX14 3DB, Abingdon, UK.
}

\begin{abstract}
:
Fusion experiments with wall cladding of different chemical elements face the problem of material mixing by the plasma-wall interaction. Understanding of experiments that investigate this long term material re-distribution require an iterative approach that takes the local erosion, the global transport of material by the scrape-off layer (SOL) plasma and the local redeposition into account. We present a model that combines parameterized DIVIMP calculations and a simplified sputtering model to simulate the time evolution of first wall material migration in a fusion experiment. The simulations are benchmarked by $\mathrm{Be}$ evaporation experiments performed previously at JET. The time evolution of the spectroscopically measured Be erosion fluxes for $800 \mathrm{~s}$ total plasma time at the main wall and inner divertor can be reproduced by the model using an extended calculation grid for DIVIMP and an increased cross field diffusivity for impurity ions in the SOL. Discrepancies were found in the outer divertor region.
\end{abstract}

Keywords:

PSI-19:

Beryllium, DIVIMP, Edge plasma, Erosion \& Deposition, Impurity transport, JET

JNM:

B0100 Beryllium, I0100 Impurities, P0500 Plasma-Materials Interaction, F0400 First Wall Materials, S1300 Surface Effects

PACS:

28.52.Fa, 52.25.Vy, 52.40.Hf, 52.65.Pp, 61.80.Jh

Corresponding Author / Presenting Author Address:

Dr. Matthias Reinelt

Max-Planck-Institut für Plasmaphysik

Materials Research Division

Boltzmannstrasse 2

85748 Garching Germany

Tel.: +49 8932991614

Fax.: +498932991212

Corresponding Author e-mail / Presenting Author e-mail:

matthias.reinelt@ipp.mpg.de 


\section{Introduction}

Fusion experiments that rely on different chemical elements as first wall materials benefit from the combination of different desired material properties at different wall segments but face the problem of material mixing, which can eventually counteract the initially intended surface properties by changing the surface composition over time. During a discharge, wall material is eroded by particles from the plasma or by thermal processes such as sublimation due to the heat loads from the plasma. If the eroded particles enter the plasma they eventually get ionized and are thus susceptible to forces in the plasma such as friction, diffusion and electromagnetic and thermal forces. This leads to a transport of the particles to other wall segments where they are again deposited. If the particles gain enough energy from the plasma, their deposition can again produce sputtered wall material (impurity and self sputtering). This cycle is iteratively repeated during each discharge. The plasma wall interaction therefore effectively transports wall material not only from erosion to deposition dominated zones, but each local flux of eroded wall material is redistributed over time in a broadened pattern across the first wall. The exact shape of the redistribution pattern is determined by the properties of the eroded material and scrape-off layer plasma (SOL). Because wall material erosion is dominated by the surface composition up to the implantation range of the ions (several nanometers), the redistribution of elements inevitably leads to a quick dynamic change of the plasma facing surface properties influencing the performance of the plasma discharge. Some critical issues that were identified in the past are recycling of fuel, co-deposition of tritiumrich layers [1], Be-W alloy formation [2, 3] or sublimation. In principle, such issues can be seen as a problem of a flux balance of different contributions [4]. If and how fast the surface properties change at a certain position along the main wall depend as a first approximation on the local influx of elements and the surface temperature. Furthermore, the total influx is balanced by the local outgoing flux of material that is sputtered (flux into plasma), sublimated (flux into plasma), thermally dissociated (change of the composition within the surface) or lost by diffusion (flux into the bulk). All local areal densities are coupled by transport of material in the SOL of the discharge. The model we developed is based on the described view of the processes and simulates the time evolution of the global surface areal densities as a set of ordinary differential equations representing for each wall segment the sum of all local flux contributions. The equations are coupled by the re-distribution of impurities by plasma transport. Basic concepts of the model were already used in $[4,5,6,7]$. In order to simplify the model we assume that the transport time of impurities in the plasma is small compared to the time scale of the wall composition dynamics and that the concentration of plasma impurities has negligible influence on the plasma properties (tracer limit). This enables one to parameterize the plasma transport thus reducing the problem to a dynamic change of the surfaces areal densities. It is therefore possible to perform self consistent simulations of the global impurity migration in a fusion experiment under particle conservation. This is especially important for long term simulations (i.e. more than one discharge) because of the iterative nature of the wall composition dynamics. A second assumption is that the total plasma facing wall is divided into two layers. A bulk layer represents a perfect source or sink for all components with a constant composition, while a thin surface layer ("reaction zone") is characterized by a constant total areal density and a homogeneous distribution of all components. Balancing fluxes derived from the total net fluxes from or to the plasma or within the reaction zone keep the total areal density of chemical phases in the reaction zone constant. The coupled set of $\mathrm{k} \cdot \mathrm{n}_{\mathrm{B}}$ ordinary differential equations for $\mathrm{n}_{\mathrm{B}}$ wall elements and $\mathrm{k}$ chemical phases are solved numerically by Mathematica 7.0 [8]. Further details of the algorithms and concepts used in the model are given in [9]. To asses the possibilities of the proposed model we apply it to beryllium evaporation experiments performed in JET [10]. 


\section{Application of the model to JET:}

The magnetic flux structure of the SOL plasma we use is based on a large SONNET [11] grid "sonnet_68124_49000.lg” adopted for JET discharge number 68124. The grid is cut with the vessel wall by newly developed DIVIMP [12] routines that provide a much closer match of the grid with the vessel wall. For comparison, the grid is also cut in a fashion that results in a grid similar to that of the previously used small grid form [10]. This grid leaves large gaps between the main wall and the last ring of grid cells, but is commonly used by plasma codes such as SOLPS (B2/Eirene) due to the rectangular data structure of the grid cells. The background plasma (pure deuterium) is generated by DIVIMP "sol28" option, an onion skin model (OSM) with particle and momentum conservation [13, 14, 15] and 6 successive iterations with Eirene07 [16]. Experimental electron temperature $\left(T_{e}\right)$ and density profiles $\left(n_{e}\right)$ from Langmuir probe data are used as constraints for the OSM in the divertor and outer mid plane. The resulting plasma emits a deuterium flux from the outer most grid cells ("ion wall”) towards the vessel wall ("neutral wall"). Depending on whether a magnetic flux surface cuts the ion wall or is parallel to the ion wall, the emitted background plasma flux is either perpendicular or parallel to the magnetic field. Perpendicular fluxes are assumed to be driven by the deuterium density gradient to adjacent cells, while parallel fluxes mainly depend on the flow velocity of the plasma [17]. Parallel fluxes are usually much larger than perpendicular fluxes. The extension of the grid leads to a distribution of parallel and perpendicular fluxes across the neutral wall. In previous models [e.g. 18] using the small grids, only fluxes at the targets were treated as parallel. In order to asses the resulting deuterium fluxes onto the main wall, the flux vectors leaving the ion wall are projected onto the neutral wall by a ray tracing like algorithm. Fig. 1a and $1 \mathrm{~b}$ shows the flux vectors emitted from the ion wall for the extended (a) and small (b) grid. It can be seen that for the extended grid parallel fluxes reach parts of the lower main wall, thus resulting in a changed flux distribution along the wall. However, because the ion wall is much closer to the neutral wall, the resulting plasma temperature of the ion wall is much lower. The increased sputtering of wall material that would result from the increased plasma wall flux for the extended grid is therefore counterbalanced by the decreased plasma temperature. The resulting kinetic energy $\mathrm{E}$ with which deuterium ions impinge on the neutral wall is assumed to be $3 \mathrm{~T}_{e}+2 \mathrm{~T}_{\mathrm{i}}$ [17], resulting in an energy near the threshold for the physical sputtering of Be and C. Therefore the resulting initial Be erosion flux is very sensitive to changes of the plasma temperature and wall flux. In these calculations, only a background flux of charged species is considered. To further include the neutral wall flux, the energy and flux distribution from EIRENE07 has to be added (not included here). However, as can be seen in fig. 2 a, the measured initial Be erosion flux (at $\mathrm{t}=$ $0 \mathrm{~s}$ ) of $1.2 \cdot 10^{18} \mathrm{~m}^{-2} \mathrm{~s}^{-1}$ at the main wall corresponds quite well to the calculated flux of $1.8 \cdot 10^{18}$ $\mathrm{m}^{-2} \mathrm{~s}^{-1}$ which is mainly determined by the measured $\mathrm{T}_{\mathrm{e}}$ profiles that are a constraint for the sol28 model.

To reduce the computational effort of the model, the neutral wall used by DIVIMP with 190 elements is binned to $n_{B}=67$ groups of wall segments reducing the resolution predominantly in the divertor region but still giving a reasonable resolution across the whole main wall. The numbered dots in fig. 1c indicate the positions of start and endpoints of bins forming the reduced neutral wall.

In the experiments, beryllium was evaporated prior to a series of identical discharges with 4 toroidal-symmetrically distributed evaporators onto the main wall. Details of the experiments are given in [10]. Because the model is only a 2 dimensional representation, we assume a toroidal linear Be source at $\mathrm{r}=3.5 \mathrm{~m}$ and $\mathrm{z}=-0.4 \mathrm{~m}$. A mass of $0.2 \mathrm{~g}$ of Be is distributed onto the wall scaled with $1 / \mathrm{d}$ with $\mathrm{d}$ as the distance of the center of the bin to the source. The resulting maximum thickness of Be is $2 \mathrm{~nm}$ near the evaporator (bin 36), which corresponds to a surface areal density of $2.7 \cdot 10^{20} \mathrm{~m}^{-2}$. A number of bins (45 to 47) at the outer divertor 
nook is assumed to be shadowed from evaporation. The bulk material is set to pure $\mathrm{C}$ for all bins. Fig. $1 \mathrm{c}$ shows the initial Be areal density color coded for all neutral wall bins.

The total areal density of the reaction zone is a free parameter of the model that results from the reduction of the real depth profiles to a 0-dimensional surface layer. Nevertheless it cannot be arbitrarily chosen because the partial erosion fluxes $\Gamma_{i}$ are determined by the relative concentration $\mathrm{C}_{\mathrm{i}}$ (areal density fraction) of a chemical element $\mathrm{i}$ in the reaction zone ( $\mathrm{Y}$ is the sputter yield of pure element $\mathrm{i}$ by $\mathrm{D}$ and $\Gamma_{D}$ is the plasma wall flux):

$$
\Gamma_{i}=C_{i} Y_{i, D}(E) \Gamma_{D}
$$

This means that the reaction zone should be in the order of magnitude of the interaction depth of the ions with the surface. If the reaction zone is too thick, the erosion of elements with a low areal density is exceedingly underestimated. Because the erosion is mainly driven by the hydrogen emitted by the background plasma, the constant total areal density of the reaction zone is determined by the range of hydrogen atoms with an average energy of about $20 \mathrm{eV}$ in a C/Be mixture, which is 3-4 $\mathrm{nm}$. Accordingly, the total areal density of the reaction zone of the model is homogeneously set to $4 \cdot 10^{20} \mathrm{~m}^{-2}$. To calculate the energy dependent physical sputter yield $Y_{i, j}(E)$ of $\mathrm{i}=\mathrm{Be}$ or $\mathrm{C}$ and $\mathrm{j}=\mathrm{D}$, Be or $\mathrm{C}$ across the neutral wall we apply the Bohdansky formula [19]. The contribution of chemical erosion of $\mathrm{C}$ is not yet implemented, although this is straight forward in the context of this approach. The current calculations are nevertheless justified at least for the main wall processes because of the low surface temperature. In some cases however, chemical erosion may add a significant $\mathrm{C}$ erosion flux to the total balance. Input parameters $E_{\text {th }}$ (sputter threshold energy) and $\mathrm{Q}$ are taken from Eckstein et. al. [20] or from parameterized static TRIM calculations [21] for missing ion/target combinations. The energy dependent physical sputter yields of pure elements are scaled linearly with the elemental concentrations in the reaction zone (Equ. 1). This yields a good approximation for the partial yields for all Be/C ratios compared to detailed TRIM calculations because of the similar masses of $\mathrm{Be}$ and $\mathrm{C}$. The resulting local erosion fluxes of $\mathrm{Be}$ and $\mathrm{C}$ are distributed onto the entire neutral wall according the pre-calculated redistribution matrices (see section 3).

The spectroscopically measured fluxes of Be [22] are assumed to correspond to the gross erosion fluxes, i.e. the sputtered flux of Be that is generated by the impinging energetic $\mathrm{D}, \mathrm{Be}$ and $\mathrm{C}$ ions from the plasma. To compare the measured erosion fluxes with the modeling, we further assume that the fluxes from all bins that are visible to the spectroscopic lines are summed up (weighted by the relative bin length) to yield the total Be erosion flux that would be measured by spectroscopy. Each spectroscopic line has therefore two contributions: One flux contribution from the wall elements near the detector and one flux contribution originating from the wall elements across the detector. Modifications of the observed impurity fluxes by the plasma after the sputtering event and Be contributions from wall elements not covered by the spectroscopic lines are neglected. Within the frame of these assumptions it is possible to directly compare the time evolution of the modeled and measured Be erosion fluxes.

\section{Results and discussion:}

In order to parameterize the impurity transport via the background plasma, a number of DIVIMP runs equal to the number of bins $\left(\mathrm{n}_{\mathrm{B}}\right)$ are started and a homogeneously distributed constant impurity flux of a selected species at each bin is launched. The angular and energy distribution of the launch is determined by the local background plasma conditions. Each DIVIMP run launches 20000 independent test particles (Monte-Carlo-approach) into the plasma and calculates the trajectories through the SOL based on the forces onto the particle. The re-distribution of test particles along the neutral wall is mapped in a $\left(\mathrm{n}_{\mathrm{B}} \times \mathrm{n}_{\mathrm{B}}\right)$ matrix and 
normalized to the initial flux and the source bin length. Fig. 2d shows a Be re-distribution matrix for the extended grid as color coded re-deposited flux fractions. The coordinates of the matrix are the bin numbers as used in fig. 1c. The matrices for Be and $C$ (not shown here) are very similar. The diagonal elements correspond to the local re-deposition of impurities, i.e. the launched test particles re-enter the neutral wall and are deposited onto the same bin they originated from. The locally re-deposited flux fraction dominates the re-distribution with an average value of 0.4. Elongated vertical patterns in fig. $2 \mathrm{~d}$ correspond to long range transport. The matrix of the small grid configuration (not shown) generally displays a broader distribution especially in the outer main wall region (bins 25 to 40) while for the extended grid configuration (fig. 2d) two broader distribution pattern can be seen at the inner and outer lower main wall (bins 8 to 15 and 42 to 45). These originate from features of the vessel wall that receive parallel fluxes in the extended grid model while they are not detailed in the SOL plasma description for the small grid, as illustrated in fig. 1a and $1 \mathrm{~b}$.

When the measured spectroscopic gross erosion fluxes along the spectroscopic lines $\mathrm{H}$ (main wall), I (inner divertor) and O (outer divertor) are compared to the modeling results (fig. 2ac), we find that the initial erosion fluxes are higher for the small than for the extended grid. The extended grid yield a better fit to the experimental data due to the lower ion wall temperature and therefore reduced local sputter yields. However, the decay time of the flux is considerably changed by the properties of the applied grid and background plasma.

The local Be gross erosion flux is determined by the plasma wall flux and the local sputter yield of Be by $\mathrm{D}$ given the local ion temperature plus the sputtering by the local impurity influx at the given ion energy. The fraction of impurity sputtering (i.e. sputtering of Be or $\mathrm{C}$ by Be and $\mathrm{C}$ ) to the total sputtered flux is lower than $10 \%$ for the background plasma used here. Most of the total eroded Be is immediately transported back by the strong diagonal component of the re-distribution matrix. That means that the integrated Be gross erosion flux over time can be much higher than the locally available Be areal density because the same Be is eroded over and over again from the surface (and locally re-deposited). The time constant of the Be gross erosion flux decay is therefore determined by how much of the eroded flux is transported away from its origin by the plasma over a distance to the next bin. That means that a broadening of the re-distribution matrix increases the time constant of the erosion flux decay.

One key parameter that has a significant influence on the width of the local re-distribution is the cross-field diffusion coefficient, which is related to the probability for a test particle to drift away from the magnetic flux surface. This parameter is usually assumed to be $1 \mathrm{~m}^{2} / \mathrm{s}$ as in the core plasma. Increasing the cross field diffusivity considerably changes the patterns to more local re-deposition. This is because the chance that a test particle performs local diffusive steps perpendicular to the field lines instead of being transported away from its origin along the field lines is increased. It has therefore also an increased chance of reaching the ion wall close to its origin. The broad features of the matrix shown in fig. $2 \mathrm{~d}$ that originate from the impact of parallel fluxes on the main wall around bins 12 and 42 disappear accordingly when the cross field diffusivity is increased. The solid lines in fig. 2a-c show the results of the model with small and extended grid and different cross-field diffusion coefficients. To study the sensitivity of the model to the cross-field diffusivity as a free parameter, 1 and $20 \mathrm{~m}^{2} / \mathrm{s}$ are compared in fig. 2a-c. The agreement between the time evolution of the measured and calculated erosion fluxes is much better for the increased diffusivity at the main wall and inner divertor. The initial erosion fluxes are not changed by this modification. However, we cannot exclude at the moment that also other parameters influence the width of the re-distribution.

The initial Be erosion flux calculated by the model at the outer divertor is too high compared to the experiment. This indicates that the background plasma model yields a plasma wall flux that is too high at this position. The rapid drop of the Be surface concentration results in a 
very steep exponential decay. At this region the plasma background has to be further optimized to fit the experimental data. Additionally, chemical erosion effects (C) and sublimation (Be) due to elevated temperatures are not yet included here. These might also have an influence on the flux balances especially in the divertor region. Inclusion of chemical effects in this modeling context is discussed in [23]. It has to be noted at this point, that given the amount of uncertain input parameters, this comparison between experiment and simulation can be performed successfully with a large number of parameter combinations. Especially the plasma parameters and transport processes very close to the first wall, which govern most of the material transport, are uncertain and nevertheless have a great influence on the observed decay times of the erosion fluxes. An additional free parameter introduced by the wall dynamics model itself is the total areal density of the reaction zone. An increase of the total areal density would also increase the decay length of the Be erosion but simultaneously decrease the initial Be erosion flux by decreasing the relative atomic fraction at the surface (at a given Be areal density). Given an eroding background flux and energy, as well as a Be surface concentration, the only free parameter is the reaction zone thickness to reproduce the observed initial Be erosion flux. Additionally, the choice for this parameter can be narrowed down by the interaction depth of the eroding species and the surface material as discussed above. To develop a self-consistent model with qualitative and quantitative predictive capabilities, more parameter studies and benchmarking especially of the SOL near the first wall are needed.

\section{Summary:}

The proposed integrated model is capable of integrating parameterized (timeless) MonteCarlo DIVIMP calculations and a simplified plasma-wall interaction model to a time dependent global model of wall composition dynamics. It includes self and impurity sputtering and can be extended by additional flux contributions such as chemical phase formations or sublimation [23]. The benchmarking of the simulations with JET measurements shows that some aspects of the experimental data can be reproduced. Discrepancies were found in the outer divertor region. Comparing the decay time of a depleting Be impurity source of the experiment and simulation we find that the long range re-distribution processes are massively influenced by the cross field diffusivity. Local re-deposition processes dominate the material migration of Be and C. 


\section{References:}

[1] J. Roth, E. Tsitrone, A. Loarte, Th. Loarer, G. Counsell, R. Neu, V. Philipps, S. Brezinsek, M. Lehnen, P. Coad, Ch. Grisolia, K. Schmid, K. Krieger, A. Kallenbach, B. Lipschultz, R. Doerner, R. Causey, V. Alimov, W. Shu, O. Ogorodnikova, A. Kirschner, G. Federici \& A. Kukushkin, J. Nucl. Mater., 390-391 (2009), 1-9.

[2] Ch. Linsmeier, K. Ertl, J. Roth, A. Wiltner, K. Schmid, F. Kost, S.R. Bhattacharyya, M. Baldwin \& R.P. Doerner, J. Nucl. Mater., 363-365 (2007), 1129-1137.

[3] F. Kost, Ch. Linsmeier, M. Oberkofler, M. Reinelt, M. Balden, A. Herrmann \& S. Lindig, J. Nucl. Mater., 390-391 (2009), 975-978.

[4] K. Krieger \& J. Roth, J. Nucl. Mater., 290-293 (2001), 107-111.

[5] K. Schmid, K. Krieger, A. Kukushkin \& A. Loarte, J. Nucl. Mater., 363-365 (2007), 674679.

[6] K. Schmid, M. J. Baldwin, R. P. Doerner \& D. Nishijima, Nucl. Techn., 159 (2007), 238244.

[7] K. Schmid, Nucl. Fusion, 48 (2008), 105004.

[8] Wolfram Research Inc., Mathematica, Version 7.0, Champaign, IL (2008).

[9] K. Schmid, these proceedings.

[10] K. Krieger, S. Brezinsek, S. Jachmich, S. Lisgo, M. Stamp, H.G. Esser, A. Kreter, S. Menmuir, Ph. Mertens, V. Philipps, P. Sundelin \& JET EFDA contributors, J. Nucl. Mater., 390-391 (2009), 110-114.

[11] R. Marchand \& M. Dumberry, Comp. Phys. Comm., 96 (1996), 232-246.

[12] P.C. Stangeby \& J.D. Elder, J. Nucl. Mater., 196-198 (1992), 258-263.

[13] S. Lisgo, P.C. Stangeby, J.D. Elder, J.A. Boedo, B.D. Bray, N.H. Brooks, M.E. Fenstermacher, M. Groth, D. Reiter, D.L. Rudakov, J.G. Watkins, W.P. West \& D.G. Whyte, J. Nucl. Mater., 337-339 (2005), 256-260.

[14] P. C. Stangeby, J. D. Elder, W. Fundamenski, A. Loarte, L. D. Horton, R. Simonini, A. Taroni, O. F. Matthews \& R. D. Monk, J. Nucl. Mater., 241-243 (1997), 358-362.

[15] S. K. Erents, P. J. Davies, J. D. Elder, H. Y. Guo, L. D. Horton, G. F. Matthews, R. D. Monk, P. C. Stangeby \& D. D. R. Summers, J. Nucl. Mater., 241-243 (1997), 433-437.

[16] A. Loarte, A. S. Kukushkin, H. D. Pacher, D. P. Coster, R. Schneider, N. Asakura, K. Itami, B. LaBombard, B. Lipschultz, C. F. Maggi, R. D. Monk, G. D. Porter, M. Shimada, M. Sugihara \& J. Terry, J. Nucl. Mater., 266-269 (1999), 1123-1128.

[17] The Plasma Boundary of Magnetic Fusion Devices, Taylor \& Francis, (2000), 1. Edition.

[18] G.F. Matthews, P.C. Stangeby, J.D. Elder, N.A.C. Gottardi, P.J. Harbour, L.D. Horton, H.J. Jackel, L. de Kock, A. Loarte, C.F. Maggi, D.P.J. O'Brien, R. Simonini, J. Spence, M.F. Stamp, P.E. Stott, H.P. Summers, J. Tagle \& M. von Hellerman, J. Nucl. Mater., 196-198 (1992), 374-379.

[19] J. Bohdansky, Nucl. Instr. and Meth.: B, 2 (1984), 587-591.

[20] Sputtering data, IPP Report, IPP 9/82, (1993).

[21] W. Eckstein \& J. Biersack, Nucl. Instr. and Meth.: B, 2 (1984), 550-554.

[22] M. Stamp, these proceedings.

[23] Ch. Linsmeier, these proceedings. 


\section{Figure captions:}

Figure 1:

The arrows in (a), extended grid, and (b), small grid, indicate the direction of the plasma wall fluxes leaving the ion wall. Gray arrows represent perpendicular fluxes, orange arrows fluxes that are parallel to the magnetic flux surfaces. Part c shows the initial Be areal density (color coded) and numbering if the bins along the vessel wall. The highest surface areal density of $2.7 \cdot 10^{20} \mathrm{~m}^{-2}$ is near the evaporation source at bin 36 . The spectroscopic lines are indicated schematically by $(\mathrm{H})$ for the main wall and $(\mathrm{I})$ and $(\mathrm{O})$ for the inner and outer divertor, respectively. The star indicates the position of the Be evaporation source.

Figure 2:

Experimental [10] and modeled time evolution of the Be erosion fluxes along the spectroscopic lines in the main wall (a), the inner divertor (b) and outer divertor region (c). The dotted and dashed lines are calculated with a re-distribution matrix based on the small (SG) and extended grid (EG) with a cross field diffusivity of $1 \mathrm{~m}^{2} \mathrm{~s}^{-1}$, the solid line is based on the extended grid and a cross field diffusivity of $20 \mathrm{~m}^{2} \mathrm{~s}^{-1}$. A re-distribution matrix calculated for Be by DIVIMP on the extended grid is shown in (d). The bin numbering corresponds to that of fig. 1c. The re-distributed Be flux fraction is color coded (truncated at 0.05). Black means 0 and white means that more than 0.05 of the flux started at a source bin is transported to the destination bin. 
Figure 1:

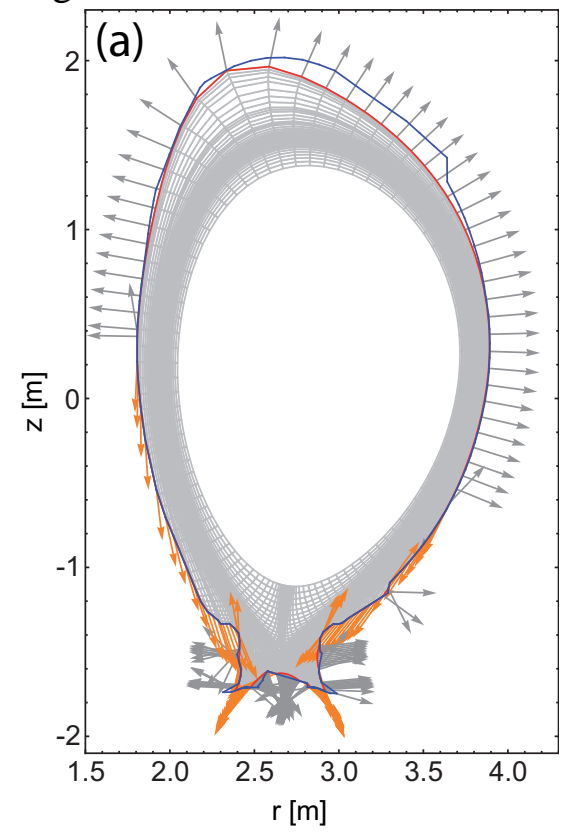

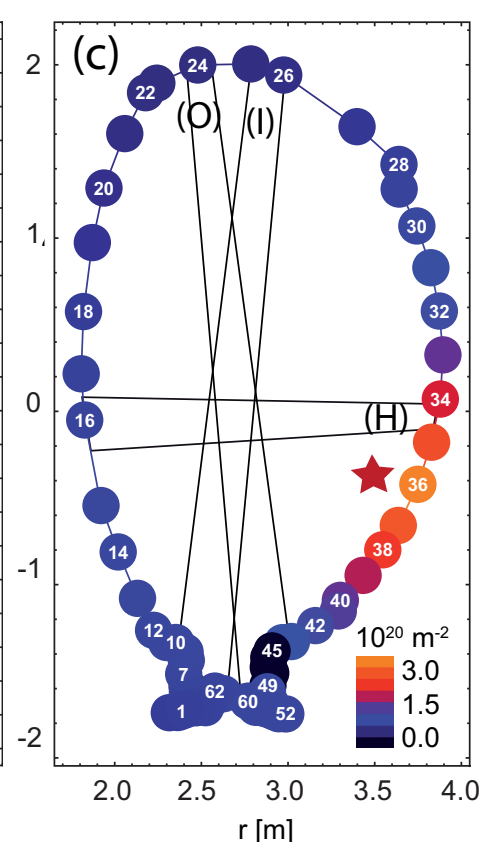


Figure 2:

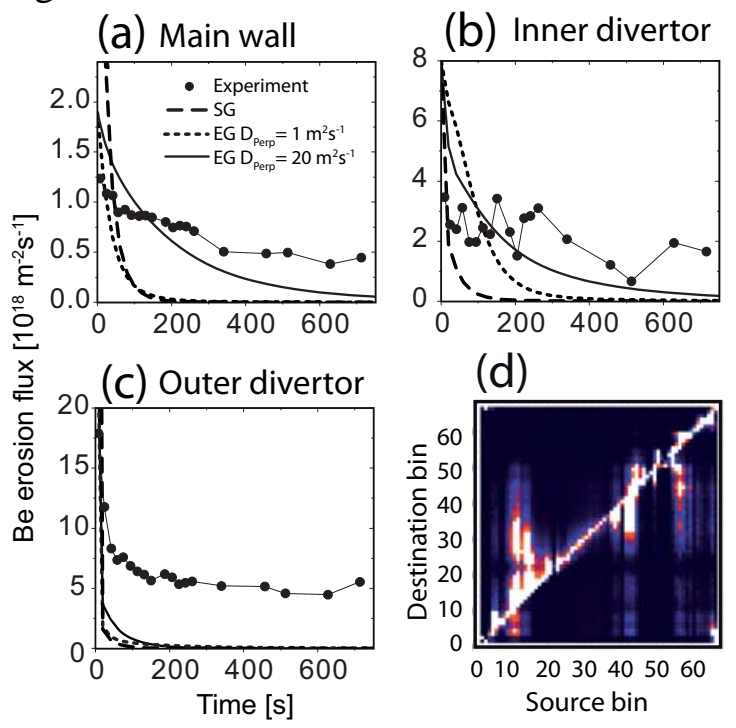

